\title{
Interacting Electrons on a Square Fermi Surface
}

\author{
A. Luther \\ NORDITA, Blegdamsvej 17, DK-2100 Copenhagen Ø, Denmark
}

(February 7, 2018)

\begin{abstract}
Electronic states near a square Fermi surface are mapped onto quantum chains. Using boson-fermion duality on the chains, the bosonic part of the interaction is isolated and diagonalized. These interactions destroy Fermi liquid behavior. Non-boson interactions are also generated by this mapping, and give rise to a new perturbation theory about the boson problem. A case with strong repulsions between parallel faces is studied and solved. There is spin-charge separation and the square Fermi surface remains square under doping. At half-filling, there is a charge gap and insulating behavior together with gapless spin excitations. This mapping appears to be a general tool for understanding the properties of interacting electrons on a square Fermi surface.
\end{abstract}

\section{INTRODUCTION}

The problem of interacting electrons in two space dimensions is thought to be central to an understanding of high temperature superconductivityl. Ordering in three dimensions, according to this scenario, arises from the coupling of highly correlated layers2. Attention naturally focusses on the properties of the layers and the possibility of unconventional behavior in two dimensions.

A standard model often studied in this context is the Hubbard model. This begins with electrons which may tunnel between nearest neighbor sites on a square lattice, with amplitude $t$, and adds an on-site repulsion of strength $U$. In comparison with the corresponding problem in one dimension $\mathrm{B}$, relatively little is known about this model.

Perturbation theory in $U$ shows very interesting and complicated behavior. With the starting point of an average occupation number of one electron per site, serious divergences are encountered 1 . These have been analyzed for more general interactions than the Hubbard model interaction $\mathbf{E}$ and are worse than the usual logarithmic type found in other problems. Evidently, there is a need for new techniques to be developed.

The starting point of one electron per site has a square Fermi surface. This paper discusses a novel mapping of the square Fermi surface onto one dimensional chains. Two sets 
of chains arise, one for each axis of the square. Interactions can be decomposed into couplings between parallel faces of the square and adjacent faces. These, in turn, result in interactions between the chains. Boson-fermion duality 6 can be used on each chain separately to find the equivalent boson problem, giving a precise definition of this duality for the square Fermi surface.

In a natural way, the interactions separate into purely bosonic terms plus other new operators. The bosonic terms can be diagonalized exactly and produce behavior similar to the Luttinger model in the sense that there are no fermion quasi-particles or Fermi liquid behaviort. There is complete separation of the charge and spin degrees of freedom, as previously found in purely one dimensional models $\mathrm{B}$. As a consequence, charge excitations have no spin and pure spin excitations no charge.

Non-bosonic terms produced by this mapping give rise to a new starting point for perturbation theory. This begins with the free electron kinetic energy plus the bosonic terms taken together as the unperturbed Hamiltonian. The non-boson terms arising in the mapping are then taken as the perturbation. It is then important to analyze the operators in this new perturbation theory, to see if they are relevant or irrelevant, with respect to the bosonic starting point.

This technique of separating interactions into a bosonic piece plus other operators is familiar from many other problems, such as the Kondo model 1 , the backward scattering model $\mathbf{l}^{\text {, or }}$ the sine-Gordon problem in 1D 10 . It is slightly more complicated here due to the mapping of the square Fermi surface onto chains.

One of the complicating features of the square Fermi surface concerns the modulation of the Fermi velocity along the face - in fact it vanishes at the corners. In this new perturbation analysis, it turns out that the operator causing modulation is irrelevant for any interaction. The large distance behavior is characterized by a constant velocity.

It might be argued that doping would destroy these results, since the Fermi surface should then depart from a square. That is only correct for nearly free electrons. For sufficiently strong interactions, the operator which causes deviations from the square becomes irrelevant. A square remains a square under doping, only the value of the Fermi momentum changes.

Generally, it is found that two different types of operators are relevant for repulsive interactions. These correspond to Umklapp terms and an antiferromagnetic spin-flip process. For a restricted case of repulsive interactions between parallel faces, these extra terms can also be diagonalized. They lead to a gap in the charge excitation spectrum and, therefore, insulating behavior. There are gapless spin excitations. This range of coupling constants also exhibits spin-charge separation.

There have been studies of coupled 1D chains using models for the coupling 1 which differ from that encountered in this paper. Consequently, the results cannot be applied here. Other studies of the square Fermi surface problem have concentrated on models with boson- 
like interaction 12 and found non-Fermi liquid behavion 13 . It is not clear how to extend these to include the non-boson interactions which are found to be relevant for short range interactions.

The techniques introduced in this paper are generally applicable for understanding the relevant degrees of freedom for interacting electrons on a square Fermi surface. Although the special case of interactions between parallel faces is emphasized, it should be easy to generalize these results to other models, including the Hubbard model. Future work will doubtlessly explore the fall possibilities of this mapping. Some interesting directions are indicated in the discussion section.

\section{MAPPING THE SQUARE FERMI SURFACE ONTO CHAINS}

The general problem of interacting electrons in two space dimensions, described by the tight binding approximation on square lattices, simplifies if attention is focused on low energy excitations near the Fermi surface. For the half-filled hand, the Fermi surface is a square, and the important excitations preserve this symmetry. Intuitively, excitations perpendicular to a face of the square can be thought of as one-dimensional in character if the momentum parallel to the face is zero. For large parallel momentum, this picture is not obvious since the Fermi velocity can vary with position along the face. In addition, low energy excitations between different faces can also exist, confusing a separation into excitations of each separate face.

These different excitations are contained in the kinetic energy operator of the tight-binding model,

$$
H_{o}=-t \sum_{\langle i j\rangle \sigma} a_{i \sigma}^{\dagger} a_{j \sigma}+\mu \sum_{i \sigma} a_{i \sigma}^{\dagger} a_{i \sigma}
$$

where $a_{i \sigma}$ is the Fermi operator of an electron at site $i$ with spin $\sigma, t$ is the hopping matrix element, and the sum over $i$ and $j$ is restricted to nearest neighbors on a square lattice. After a Fourier transformation, and rotation by $45^{\circ}$, this can be written as:

$$
H_{o}=-4 t \sum_{\vec{k} \sigma} \cos \left(k_{x} s^{\prime}\right) \cos \left(k_{y} s^{\prime}\right) a_{\vec{k}, \sigma}^{\dagger} a_{\vec{k}, \sigma}
$$

where $s^{\prime}=s / \sqrt{2}$. The fermion field operator is defined before rotation as $N^{-1} \sum_{\vec{k}^{\prime}} a_{\vec{k}^{\prime}, \sigma} e^{i \vec{k}^{\prime} \cdot \vec{R}^{\prime}}$, for a lattice of $N^{2}$ sites, area $L^{2}$, lattice constant "s", and $\vec{k}^{\prime}=2 \pi L^{-1}\left(n_{2}^{\prime}, n_{y}^{\prime}\right)$. The sum is the over over the Brillouin zone, and the chemical potential $\mu$, in Eq. (2.1) is chosen such that the energy of Eq. (2.2) vanishes for $k_{x}$ or $k_{y}$ equal to $\pm k_{F}$, with $k_{F}=\pi(\sqrt{2} s)^{-1}$. This defines the square Fermi surface.

Consider now that face for $k_{x}$ near $k_{F}$. The energy can be linearized to find

$$
E_{1}\left(k_{x}\right)=v_{F}\left(k_{y}\right)\left(k_{x}-k_{F}\right)
$$


where $v_{F}\left(k_{y}\right)=(2 \sqrt{2} t s) \cos \left(k_{y} s^{\prime}\right)$ and the "1" subscript refers to the particular face with $k_{x}=k_{F},-k_{F}<k_{y} \leq k_{F}$, and positive (or zero) Fermi velocity. There is a similar linearization for the other three faces, labelled by " 2 ", with $k_{x}=-k_{F}$, "3" with $k_{y}=k_{F}$, and "4" with $k_{y}=-k_{F}$, in all cases with positive (or zero) Fermi velocity.

For a given spin, the Fermi operator describing the "1" face is given, after rotation, by

$$
\psi_{1}^{\prime}(x, y)=\frac{1}{N} \sum_{k_{y}, k_{x}} a_{1 k} e^{i k_{y} y+i k_{x} x}
$$

The $\vec{k}$ momenta are $45^{\circ}$ rotations of the $\vec{k}^{\prime}, k_{y}$ runs from $-k_{F}$ to $k_{F}, x$ and $y$ are rotated coordinates. With $\vec{R}^{\prime}=s(m, n)$, these are given by $\vec{R}=(x, y)$ with $\sqrt{2} x=s(m+n)$ and $\sqrt{2} y=s(n-m)$. The equal time correlation function $\left\langle\psi_{1}^{\prime \dagger}(x, y) \psi_{1}^{\prime}\right\rangle$ is given by

$$
\begin{aligned}
\left\langle\psi_{1}^{\prime \dagger}(x, y) \psi_{1}^{\prime}\right\rangle & =s^{2} \int_{-k_{F}}^{k_{F}} \frac{d k_{y}}{2 \pi} e^{-i k_{y} y} \int_{-\infty}^{k_{F}} \frac{d k_{x}}{2 \pi} e^{-i k_{x} x-\alpha\left|k_{x}-k_{F}\right|} \\
& =\pi k_{F}^{-1} g\left(l-l^{\prime}\right)\left(\frac{i e^{-i k_{F} x}}{2 \pi(x+i \alpha)}\right)
\end{aligned}
$$

where $g(l)=2^{-1} \delta_{l, o}+(\pi l)^{-1} \sin \left(\pi l 2^{-1}\right)$, with $l=n-m$ representing a discrete "y" coordinate, and $\alpha$ is a cutoff of order $k_{F}^{-1}$ for states far away from the Fermi surface. In these formula the sums over $k$ have been replaced by integrals, using the invariance of $d k_{x} d k_{y}$ under rotation.

This result is a product of a function of the $y$ variable times a purely $1 \mathrm{D}$ expectation value. It suggests that the Fermi operator describing excitations near the "1" face can be written in the form:

$$
\psi_{1}^{\prime}(x, y)=\sqrt{\frac{\pi}{k_{F}}} \sum_{l^{\prime}}^{2 N} g\left(l-l^{\prime}\right) \psi_{1 l^{\prime}}(x)
$$

where the prime denotes the operator for the square Fermi surface, the operator on the right of Eq. (2.6) describes fermions on independent chains, and $\sqrt{2} y=s l$. The integer $l$ runs from 1 to $2 N$, since $l=n-m$ with $m$ and $n$ running from 1 to $N$.

The expectation value for independent chains $\left.\left\langle\psi_{1 l_{1}}^{\dagger}(x) \psi_{1 l_{2}}\right)\right\rangle$ with normalization $\psi_{l}(x)=$ $L^{-\frac{1}{2}} \sum_{k_{x}} a_{l}\left(k_{x}\right) e^{i k_{x} x}$, is:

$$
\begin{gathered}
\left\langle\psi_{1 l_{1}}^{\dagger}(x) \psi_{1 l_{2}}\right\rangle \delta_{l_{1} l_{2}} L^{-1} \sum_{k<k_{F}} e^{-i k x} \\
=\delta_{l_{1}, l_{2}}\left(\frac{i e^{-i k_{F} x}}{2 \pi(x+i \alpha)}\right)
\end{gathered}
$$


which gives Eq. (2.5) when $\sum_{l_{1}} g\left(l-l_{1}\right) g\left(l_{1}-l^{\prime}\right)=g\left(l-l^{\prime}\right)$ is used with Eq. (2.6).

It is also interesting to consider the mapping of Eq. (2.6) in the language of Fourier transforms. For $2 N$ independent chains, the transformed operator is

$$
\psi_{1 n_{y}}(x)=(2 N)^{-1 / 2} \sum_{l=1}^{2 N} \psi_{1 l}(x) e^{i p_{y} l}
$$

with $p_{y}=2 \pi n_{y}(2 N)^{-1}$ and $n_{y}$ in the interval $-N$ to $+N$. This corresponds to the interval in $k_{y}$ from $-2 k_{F}$ to $+2 k_{F}$, since $2 k_{F} y=\pi l$. The discretization of this interval is consistent with the spacing of lines of equal $k_{y}$, which is $2 \pi L^{-1} 2^{-\frac{1}{2}}$, and the phase factor $k_{y} y=n_{y}\left(2 \pi L^{-1} 2^{-\frac{1}{2}}\right) y=p_{y} l$. The point with $k_{y}=k_{F}$ has $n_{y}=\frac{N}{2}$, and the square Fermi surface thus corresponds to $2 N$ independent $1 D$ chains with the Fourier transform momentum restricted to the half-interval.

An obvious question with this mapping onto independent chains concerns the continuum limit used in the normalization of Eq. (2.7), contrasted to that of Eq. (2.4). They differ by $\sqrt{s}$, which is to be expected for a purely $1 \mathrm{D}$ system 14 . For the discrete lattice system in $1 \mathrm{D}$, the free fermion expectation value $\left\langle\psi_{1}^{\dagger}(m) \psi_{1}(o)\right\rangle$ behaves as $m^{-1}$ for large $m$, while $\left\langle\psi_{1}^{\dagger}(x) \psi_{1}(o)\right\rangle$ for the continuum model behaves as $x^{-1}$. Since $x \rightarrow m s$, the operators must differ by the multiplicative factor of $\sqrt{s}$. With the convention used in Eq. (2.7), the correlation functions are correctly reproduced by Eq. (2.6), and that is used in this paper.

This continuum limit cannot be applied to the other dimension, since the Fermi surface degeneracy occurs over a finite range in momentum space. The continuum limit can only be taken for states perpendicular to the Fermi surface, while the other direction remains discrete.

Similar considerations apply to the construction of the Fermi operator for $k_{x}$ near $-k_{F}$, the "2" states. The corresponding expectation value is found to be:

$$
\left\langle\psi_{2}^{\prime \dagger}(x, y) \psi_{2}^{\prime}\right\rangle=\pi k_{F}^{-1} g\left(l-l^{\prime}\right) \frac{i e^{i k_{F} x}}{2 \pi(-x+i \alpha)}
$$

and the $\psi_{2}^{\prime}$ field is represented by

$$
\psi_{2}^{\prime}(x, y)=\pi k_{F}^{-1} \sum_{l^{\prime}=1}^{2 N} g\left(l-l^{\prime}\right) \psi_{2 l^{\prime}}(x)
$$

where $\psi_{2 l}(x)$ is the operator for the "2" fermion on chain $l$.

The operator content of this mapping is clear. With the Fourier transform operator on the Fermi surface defined by

$$
\psi_{1 n_{y}}^{\prime}(x)=\psi_{1 n_{y}}(x),-\frac{N}{2} \leq n_{y} \leq \frac{N}{2}
$$

and: 


$$
\psi_{1 n_{y}}(x)=(2 N)^{-\frac{1}{2}} \sum_{l=1}^{2 N} \psi_{1 l}(x) e^{-i p_{y} l}-N<n_{y}<N
$$

It is seen that the Fermi surface operator is just the chain operator restricted to half the transverse Brillouin zone. This mapping is useful because interactions between the various faces of the Fermi surface have simple representations when expressed in terms of the chain picture. This is discussed in the following section.

Coupling between the chains could arise from the dependence of the Fermi velocity on the momentum along the face. This coupling is contained in the $k_{y}$ Fourier transform of Eq. (2.2) and Eq. (2.3):

$$
H_{1}=\sum_{n_{y}=-\frac{N}{2}}^{N / 2} \int_{o}^{L} \frac{d x}{i} v_{F}\left(p_{y}\right) \psi_{1 n_{y}}^{\prime \dagger}(x) \frac{\partial}{\partial x} \psi_{1 n_{y}}^{\prime}(x)
$$

Since the sum in this equation is restricted to the half interval, the $\psi_{1}$ operators are equal to the unprimed operators, and the inverse Fourier transform of Eq. (2.4) can be used to find

$$
H_{1}=\sum_{l, l^{\prime}} \int \frac{d x}{i} t_{l, l^{\prime}} \psi_{1 l}^{\dagger}(x) \frac{\partial}{\partial x} \psi_{1 l^{\prime}}(x)
$$

where

$$
t_{l, l^{\prime}}=(2 N)^{-1} \sum_{n_{y}=-\frac{N}{2}}^{\frac{N}{2}} v_{F}\left(p_{y}\right) e^{i p_{y}\left(l-l^{\prime}\right)}
$$

In the following section, it is shown that the off-diagonal elements of $t_{l, l^{\prime}}$ are irrelevant operators when interactions are included. The diagonal element represents an asymptotic Fermi velocity, $v_{o}$, of magnitude

$$
v_{o}=\frac{(\sqrt{2} t s)}{\pi}
$$

and $t_{l, l^{\prime}} \rightarrow v_{o} \delta_{l, l^{\prime}}$

Keeping only the diagonal element, the kinetic energy is a sum of independent chains. Each chain can be separately bosonized, giving the result:

$$
H_{1}=\pi v_{o} \sum_{l=1}^{2 N} \int_{o}^{L} d x \rho_{1 l}(x) \rho_{1 l}(x)
$$

where $\rho_{1 l}(x)=\psi_{1 l}^{\dagger}(x) \psi_{1 l}(x)$, and the product is normal ordered. The Fourier transforms of $\rho_{1 l}(x)$ are:

$$
\begin{aligned}
& \rho_{1}(\vec{n})=\sum_{l=1}^{2 N} \int_{o}^{L} d x e^{i p_{y} l+i k_{x} x} \rho_{1 l}(x) \\
& \rho_{1 l}(x)=(2 N L)^{-1} \sum_{n_{y} n_{x}} e^{-i k_{x} x-i p_{y} l} \rho_{1}(\vec{n})
\end{aligned}
$$


With these relations, the kinetic energy for face "1" can be written as:

$$
H_{1}=\frac{\pi v_{o}}{2 N L} \sum_{\vec{n}} \rho_{1}(\vec{n}) \rho_{1}(-\vec{n})
$$

where $n_{y}$ runs from $-N$ to $N$ and the sums over $n_{x}$ are to be cutoff as is the $1 \mathrm{D}$ case.

The commutator of $\psi_{1 l}^{\prime}(x)$, from Eq. (2.6), with the kinetic energy is

$$
\left[\psi_{1 l}(x), H_{1}\right]=i v_{o} \frac{\partial}{\partial x} \psi_{1 l}(x)
$$

using either Eq. (2.12) or Eq. (2.15) for $H_{1}$, as a result of boson-fermion for the $1 \mathrm{D}$ system on chain $l$.

These steps can be repeated for the other faces of the Fermi surface, including spin, giving the total kinetic operator (under the assumption that $t_{l, l^{\prime}} \rightarrow v_{o} \delta_{l, l^{\prime}}$ ) as:

$$
H_{o}=\frac{\pi v_{o}}{2 N L} \sum_{\vec{n}, \sigma, \beta} \rho_{\beta \sigma}(\vec{n}) \rho_{\beta \sigma}(-\vec{n})
$$

where the products are normal ordered, $\sigma= \pm 1$ is a spin index, and $\beta=1$ to 4 corresponding to the four faces.

The commutation relations are given by

$$
\begin{aligned}
& {\left[\rho_{1 \sigma^{\prime}}\left(\vec{n}^{\prime}\right), \rho_{1 \sigma}(\vec{n})\right]=-\left[\rho_{2 \sigma}\left(\vec{n}^{\prime}\right), \rho_{2 \sigma}(\vec{n})\right]=2 N n_{x} \delta_{\vec{n},-\vec{n}^{\prime}} \delta_{\sigma, \sigma^{\prime}}} \\
& {\left[\rho_{3 \sigma^{\prime}}\left(\vec{n}^{\prime}\right), \rho_{3 \sigma}(\vec{n})\right]=-\left[\rho_{4 \sigma^{\prime}}\left(\vec{n}^{\prime}\right), \rho_{4 \sigma}(\vec{n})\right]=2 N n_{y} \delta_{\vec{n},-\vec{n}^{\prime}} \delta_{\sigma, \sigma^{\prime}}}
\end{aligned}
$$

With all other commutations vanishing. Fermi operators for the various faces are:

$$
\begin{aligned}
\psi_{\beta, \sigma}^{\prime}(x, y) & =\sqrt{\frac{\pi}{k_{F}}} \sum_{l^{\prime}=1}^{2 N} g\left(l-l^{\prime}\right) \psi_{l^{\prime}, \beta, \sigma}(x) \\
\psi_{\beta^{\prime}, \sigma}^{\prime}(x, y) & =\sqrt{\frac{\pi}{k_{F}}} \sum_{k^{\prime}=1}^{2 N} g\left(k-k^{\prime}\right) \psi_{k^{\prime}, \beta^{\prime}, \sigma}(y)
\end{aligned}
$$

where $\sigma$ is the spin index, $\beta=1,2$ the face index, $l$ is the discrete coordinate corresponding to $y, \sqrt{2} y=s l$, and $x$ the continuum variable on chain $l^{\prime}$. The other two faces are rotated versions of the above, with $\beta^{\prime}=3,4$ the face index, $k$ the discrete $x$ coordinate, and $y$ the continuum variable on chain $k^{\prime}$.

The necessity to construct fermion operators unsymmetrically in $x$ and $y$ follows from the degeneracies over finite regions in momentum space, the flat pieces of the Fermi surface. The mapping onto independent chains gives the correct multiple point fermion expectation values, since it preserves the correct operator structure. This construction therefore gives the correct asymptotic behavior of these correlation functions and the dynamics near the Fermi surface. It is particularly useful when interactions between the different faces are included as described in the following section. It should be emphasized that no approximation has been made beyond linearization of the free electron spectrum at the Fermi surface and the assumption that $t_{l l^{\prime}}$ is diagonal, as discussed in Section IV. 


\section{DENSITY OPERATORS FOR THE SQUARE FERMI SURFACE}

The interactions between electron are often described by the Hubbard model,

$$
H=H_{o}+U \sum_{i} n_{i+} n_{i-}
$$

where $H_{o}$ is given by Eq. (2.1), $U$ is the on-site repulsion between electrons of opposite spins, and $n_{i \sigma}$ the number operator at site $i$ with spin $\sigma$. The previous section presented a mapping of $H_{o}$ onto independent chains, and it is natural to understand the interaction term in this representation. Basic to this construction is the assumption that states near the Fermi surface are important, since these are correctly reproduced by that mapping.

In principle, the procedure to follow is straightforward. An electron operator is just the sum of the four operators, $\psi=\psi_{1}+\psi_{2}+\psi_{3}+\psi_{4}$, from the four faces. Each can be mapped into independent chains, with two orthogonal sets of chains for the two axes of the square. Since $n_{i+}=\psi_{+}^{\dagger} \psi_{+}$, there are 256 terms, but there are also great simplifications due to symmetry and operator dimension.

The first simplification occurs in the density operators themselves. Consider the contribution to the spin $(+1)$ density from face " 1 ", using the mapping of Eq. (2.19)

$$
\begin{array}{r}
\psi_{1+}^{\prime \dagger}(x, y) \psi_{1+}^{\prime}(x, y)= \\
\pi k_{F}^{-1} \sum_{l_{1} l_{2}} g\left(l-l_{1}\right) g\left(l-l_{2}\right) \psi_{1 l_{1}+}^{\dagger}(x) \psi_{1 l_{2}+}(x)
\end{array}
$$

The terms with $l_{1}=l_{2}$ gives a purely bosonic contribution, since $\psi_{1 l_{1}+}^{\dagger}(x) \psi_{1 l_{1}+}(x)=\rho_{1 l_{1}+}(x)$ is of the form discussed in Eq. (2.16). Defining this boson piece by $\rho_{1+}^{\prime}(x, y)$, results in:

$$
\rho_{1+}^{\prime}(x, y)=\pi k_{F}^{-1} \sum_{l_{1}} g^{2}\left(l-l_{1}\right) \rho_{1 l_{1}+}(x)
$$

and has the Fourier transform, with conventions from Eq. (2.16), given by $\pi k_{F}^{-1} \rho_{1+}^{\prime}(\vec{n})$, with

$$
\rho_{1+}^{\prime}(\vec{n})=f\left(n_{y}\right) \rho_{1+}(\vec{n})
$$

In this equation, $f\left(n_{y}\right)$ is the Fourier transform of $g^{2}(l)$,

$$
f\left(n_{y}\right)=\sum_{l=1}^{2 N} e^{i p_{y} l} g^{2}(l)=\frac{1}{2}\left(1-\frac{\left|n_{y}\right|}{N}\right)=\frac{1}{2}\left(1-\frac{\left|p_{y}\right|}{\pi}\right)
$$

for $\left|n_{y}\right| \leq N,\left(\left|p_{y}\right| \leq \pi\right)$. Using the commutation relations of the $\rho_{\alpha, \sigma}(\vec{n})$ operators on the right hand side of Eq. (3.4), given in Eq. (2.17), the operator algebra for the boson part of the Fermi surface density operator can be found.

It is interesting to study the equal-time density-density correlation function, for free particles, and determine how much is contained in the purely bosonic piece of Eq. (3.4). The correlation function is found using Eq. (2.6), 


$$
\begin{aligned}
& \left\langle\psi_{1}^{\prime \dagger}(x, y) \psi_{1}^{\prime}(x, y) \psi_{1}^{\prime \dagger} \psi_{1}^{\prime}\right\rangle \\
= & \left\langle\psi_{1}^{\prime}(x, y) \psi_{1}^{\prime}\right\rangle\left\langle\psi_{1}^{\prime}(x, y) \psi_{1}^{\prime \dagger}\right\rangle \\
= & \pi^{2} k_{F}^{-2} g^{2}(l)\left(\frac{i}{2 \pi} \frac{1}{x+i \alpha}\right)^{2}
\end{aligned}
$$

while the boson part of the density operator contributes:

$$
\left\langle\rho_{1}^{\prime}(x, y) \rho_{1}^{\prime}\right\rangle=\pi^{2} k_{F}^{-2} \sum_{l^{\prime}} g^{2}\left(l-l^{\prime}\right) g^{2}\left(l^{\prime}\right)\left(\frac{i}{2 \pi} \frac{1}{x+i \alpha}\right)^{2}
$$

The sum over $l^{\prime}$ gives $\left(2 \pi^{2} l^{2}\right)^{-1}+(4)^{-1} \delta_{l, o}$, with $g^{2}(l)$ from Eq. (2.5). The difference arises from the restriction to equal chains contained in $\rho_{1}^{\prime}(x, y)$. In general, contributions form unequal chains must be examined, and if relevant, they should be retained. Nonetheless, a purely bosonic piece can always be extracted from a density operator. The unequal chain terms are analyzed in the next section.

The boson contribution to the Hubbard model interaction of Eq. (3.1) can be extracted using this procedure. After the notation used in Eq. (2.2), the contribution from the "1" face is given by

$$
\begin{aligned}
U \sum_{i} n_{i+} n_{i-} & \rightarrow U \int \frac{d x}{s} \sum_{l} \rho_{1 l+}^{\prime}(x) \rho_{1 l-}^{\prime}(x) \\
& =\frac{U}{s} \pi^{2} k_{F}^{-2} \frac{1}{2 N L} \sum_{\vec{n}} f^{2}\left(n_{y}\right) \rho_{1+}(\vec{n}) \rho_{1-}(-\vec{n}) \\
& =\frac{(U s)}{N L} \sum_{\vec{n}} f^{2}\left(n_{y}\right) \rho_{1+}(\vec{n}) \rho_{1-}(-\vec{n})
\end{aligned}
$$

Note that the combination $U s$ enters here, and this defines the coupling constant for the continuum limit, $U s$. In the solutions discussed below, only the ratio $U s / v_{o}$ appears, and from Eq. (2.12), the lattice constant cancels, as in the 1D Hubbard model continuum limit. An interaction which exists only at one site can be generalized to have different interactions strengths between different faces. By symmetry, one expects three different interactions, equal faces, opposite faces, and adjacent faces. There is also a complete separation of charge and spin degrees of freedom in these interactions, giving six coupling constants in all.

Charge-spin separation follows from choosing linear combination in the form

$$
\begin{aligned}
& \sqrt{2} \rho_{\alpha}(\vec{n})=\rho_{\alpha+}(\vec{n})+\rho_{\alpha-}(\vec{n}) \\
& \sqrt{2} \sigma_{\alpha}(\vec{n})=\rho_{\alpha+}(\vec{n})-\rho_{\alpha-}(\vec{n})
\end{aligned}
$$

where $\alpha$ is the face index. These operators satisfy

$$
\left[\sigma_{\alpha^{\prime}}\left(\vec{n}^{\prime}\right), \sigma_{\alpha}(\vec{n})\right]=\left[\rho_{\alpha^{\prime}}\left(\vec{n}^{\prime}\right), \rho_{\alpha}(\vec{n})\right]=\delta_{\alpha, \alpha^{\prime}} \delta_{\vec{n}-\vec{n}^{\prime}} 2 N\left(\hat{\epsilon}_{\alpha} \cdot \vec{n}\right)
$$


where $\hat{\epsilon}_{1}=\hat{x}, \hat{\epsilon}_{2}=-\hat{x}, \hat{\epsilon}_{3}=+\hat{y}$, and $\hat{\epsilon}_{4}=-\hat{y}$, and all other commutators vanish. An interaction term of the form in Eq. (3.7) can be re-written, using

$$
\begin{array}{r}
2 \sum_{\vec{n}, \alpha \beta} f_{\alpha \beta}(\vec{n}) \rho_{\alpha+}(\vec{n}) \rho_{\beta-}(-\vec{n}) \\
=\sum_{\vec{n}, \alpha \beta} f_{\alpha \beta}(\vec{n})\left[\rho_{\alpha}(\vec{n}) \rho_{\beta}(-\vec{n})-\sigma_{\alpha}(\vec{n}) \sigma_{\beta}(-\vec{n})\right]
\end{array}
$$

provided $f_{\alpha \beta}$ is even in $\vec{n}$ and symmetric under interchange of $\alpha$ and $\beta$, which is the case here.

The most general interaction allowed by this construction is then

$$
H_{B}=\frac{1}{N L} \sum_{\vec{n}, \alpha \beta}\left[\rho_{\alpha}^{\prime}(\vec{n}) A_{\alpha \beta} \rho_{\beta}^{\prime}(-\vec{n})-\sigma_{\alpha}^{\prime}(\vec{n}) B_{\alpha \beta} \sigma_{\beta}^{\prime}(-\vec{n})\right]
$$

with $A_{\alpha \alpha}=V_{1}$ and $B_{\alpha \alpha}=U_{1}$ representing scattering on the same face; $A_{12}=A_{21}=A_{34}=$ $A_{43}=V_{2}$ and $B_{12}=B_{21}=B_{34}=B_{43}=U_{2}$ for opposite faces, while all other elements of $A_{\alpha \beta}=V_{3}$ and $B_{\alpha \beta}=U_{3}$, representing scattering from adjacent faces, are equal. In a similar fashion, the boson form of the kinetic energy, Eq. (2.19), can be separated into spin and charge operators, using Eq. (3.8). The result is

$$
H_{o}=\frac{\pi v_{o}}{N L} \sum_{\vec{n} \alpha}\left[\rho_{\alpha}(\vec{n}) \rho_{\alpha}(-\vec{n})+\sigma_{\alpha}(\vec{n}) \sigma_{\alpha}(-\vec{n}]\right.
$$

and together with Eq. (3.10), provides a complete description of the bosonic degree of freedom for the square Fermi surface. This provides a starting point from which the relevant (or irrelevant) operators are to be determined.

For the Hubbard model, all of the six coupling constants are equal, with magnitude $2 U s$. This equality is not obviously useful for they may renormalize in different ways when relevant operators, coming from non-boson terms in the interaction, are included. Although the boson Hamiltonian $H_{o}+H_{B}$ from Eq. (3.10) and Eq. (3.11) can be diagonalized, the problem is simplified somewhat if coupling between adjacent faces is omitted, $V_{3}=U_{3}=0$, the procedure followed below.

The parameters describing boson interactions between parallel faces can be more intuitively understood by inverting the spin-charge separation. In this way, $H_{B}$ for parallel face interactions can also be written in the form:

$$
\begin{aligned}
H_{B}= & \frac{1}{2 N L} \sum_{\vec{n} \alpha \sigma \sigma^{\prime}} f^{2}\left(n_{y}\right) \rho_{\alpha \sigma}(\vec{n})\left[\left(V_{1}-U_{1}\right) \delta_{\sigma, \sigma^{\prime}}+\left(V_{1}+U_{1}\right) \delta_{\sigma,-\sigma^{\prime}}\right] \rho_{\alpha \sigma^{\prime}(-\vec{n})} \\
& +\frac{1}{N L} \sum_{\vec{n} \sigma, \sigma^{\prime}} f^{2}\left(n_{y}\right) \rho_{1 \sigma}(\vec{n})\left[\left(V_{2}-U_{2}\right) \delta_{\sigma, \sigma^{\prime}}+\left(V_{2}+U_{2}\right) \delta_{\sigma,-\sigma^{\prime}}\right] \rho_{2 \sigma^{\prime}}(-\vec{n}) \\
& +(3-4 \text { terms })
\end{aligned}
$$


where $\alpha=1,2$. The contribution from the " 3 " and " 4 " faces is found by replacing $f\left(n_{y}\right)$ by $f\left(n_{x}\right), \rho_{1}$ by $\rho_{3}$, and $\rho_{2}$ by $\rho_{4}$.

All of these are forward scattering processes. The first term has small momentum transfer on the same face, while the second describes small momentum transfer on opposite faces. Although this representation makes a physical interpretation more evident, solutions are more conveniently found using the separation into spin and charge operators.

It should also be recognized that the generalized model with six coupling constants is not the most general, even for the boson degrees of freedom. In order to have these unequal, it is necessary to have a momentum dependent interaction, rather than the on-site interaction of the Hubbard model. As a result, the momentum dependence along the square faces would also appear, which changes the momentum dependence contained in the $f(n)$ factor appearing in Eq. (3.4). The model which retains only $V_{1}, V_{2}, U_{2}$ and $U_{2}$, discussed in the following section, not only provides insight into the more general problem, but perhaps can serve as a phenomenological description as well.

\section{RELEVANT OPERATORS AND THE BOSON PROBLEM}

The preceding section discussed the construction of boson operators which are contained in the fermion density operators, and showed how to extract products of boson operators from the Hubbard interaction. In this section, the boson problem is diagonalized, and the remaining terms contained in the interaction, are isolated and discussed.

Central issues concern the value of the single electron states and the possible breakdown of Fermi liquid theory. The conditions for spin-charge separation need to be analyzed, along with the nature of the excitation spectrum. Further questions involve the consequences of the modulation of the Fermi velocity along the face and the stability of the square Fermi surface to doping away from half-filling. These problems can all be studied using the boson Hamiltonian, from the proceeding section.

The starting point for the boson problem is $H_{o}+H_{B}$, given by Eq. (3.10) and Eq. (3.11), which can be written as:

$$
\begin{aligned}
H_{o}+H_{B}= & \frac{\pi v_{o}}{N L} \sum_{\vec{n}, \alpha}\left\{\left(1+V_{1} \frac{f_{\alpha}^{2}(\vec{n})}{\pi v_{o}}\right) \rho_{\alpha}(\vec{n}) \rho_{\alpha}(-\vec{n})+\left(1-U_{1} \frac{f_{\alpha}^{2}(\vec{n})}{\pi v_{o}}\right) \sigma_{\alpha}(\vec{n}) \sigma_{\alpha}(\vec{n})\right. \\
& +\frac{2 V_{2}}{\pi v_{o}} f^{2}\left(n_{y}\right) \rho_{1}(n) \rho_{2}(-n)-\frac{2 U_{2}}{\pi v_{o}} f^{2}\left(n_{y}\right) \sigma_{1}(\vec{n}) \sigma_{2}(-(\vec{n}) \\
& \left.+\frac{2 V_{2}}{\pi v_{o}} f_{3}^{2}\left(n_{x}\right) \rho_{3}(n) \rho_{3}(-\vec{n})-\frac{2 U_{2}}{\pi v_{o}} f^{2}\left(n_{x}\right) \sigma_{4}(\vec{n}) \sigma_{4}(-\vec{n})\right\}
\end{aligned}
$$

Here the sum over $\vec{n}$ is defined from $-N$ to $N$ for momentum parallel to the face in question, with an exponential cutoff perpendicular to the face, as in the $1 \mathrm{D}$ problem. The function 
$f_{\alpha}(\vec{n})$ equals $f\left(n_{y}\right)$ for $\alpha=1,2$ and $f\left(n_{x}\right)$ for $\alpha=3,4$. Note that the coupling constant enters as the ratio $V_{1} / v_{o}$ etc., and the lattice constant contained in these objects cancels. Terms containing a zero in the denominator are to be omitted. They corresponds to zero modes, containing no space-time dependence, and must be treated separately.

Separation into spin and charge operators is explicit, and each sector can be diagonalized separately, using canonical transformations such as:

$$
\begin{aligned}
& \rho_{1}^{\prime}(\vec{n})=\cosh \eta\left(n_{y}\right) \rho_{1}(\vec{n})-\sinh \eta\left(n_{y}\right) \rho_{2}(\vec{n}) \\
& \rho_{2}^{\prime}(\vec{n})=\cosh \eta\left(n_{y}\right) \rho_{2}(\vec{n})-\sinh \eta\left(n_{y}\right) \rho_{1}(\vec{n})
\end{aligned}
$$

generated by $\rho_{1}^{\prime}(\vec{n})=e^{S_{1}} \rho_{1}(\vec{n}) e^{-S_{1}}$ with $S_{1}=(2 N)^{-1} \sum_{\vec{n}} n_{x}^{-1} \eta\left(n_{y}\right) \rho_{1}(\vec{n}) \rho_{2}(-\vec{n})$. A similar transformation diagonalizes the spin degrees of freedom for the 1-2 faces, with $S_{2}=$ $\left(2 N^{-1}\right) \sum_{\vec{n}} n_{x}^{-1} \mu\left(n_{y}\right) \sigma_{1}(\vec{n}) \sigma_{2}(-\vec{n})$. The transformation for the 3-4 faces are found by interchanging $n_{x}$ and $n_{y}$, with $1 \rightarrow 3$ and $2 \rightarrow 4$ for the boson subscripts.

Applying these transformations to Eq. (4.1) leads to the diagonal form

$$
H_{D}=\frac{\pi v_{o}}{N L} \sum_{\vec{n}, \alpha}\left[\gamma_{\alpha}(\vec{n}) \rho_{\alpha}(\vec{n}) \rho_{\alpha}(-\vec{n})+\delta_{\alpha}(\vec{n}) \sigma_{\alpha}(\vec{n}) \sigma_{\alpha}(-\vec{n})\right]
$$

where $\alpha=1,2,3,4$. The charge velocity renormalizations are given by:

$$
\gamma_{1}^{2}(\vec{n})=\gamma_{2}^{2}(\vec{n})=\left(1+\frac{V_{1}}{\pi v_{o}} f^{2}\left(n_{y}\right)\right)^{2}-\left(\frac{V_{2} f^{2}\left(n_{y}\right)}{\pi v_{o}}\right)^{2}
$$

with $\gamma_{3}(\vec{n})=\gamma_{4}(\vec{n})$ found from Eq. (4.4) by replacing $n_{y}$ by $n_{x}$. The spin velocities, $\delta_{\alpha}(\vec{n})$, are given by $\gamma_{\alpha}(\vec{n})$ with the replacements $V_{1} \rightarrow-U_{1}$ and $V_{2} \rightarrow-U_{2}$. In general, the spin and charge velocities are different, but preserve the $x-y$ symmetry.

The parameters used in these diagonalization transformations $S_{1}$ to $S_{4}$ are given by

$$
e^{-4 \eta\left(n_{y}\right)}=\frac{1+\left(\frac{V_{1}-V_{2}}{\pi v_{o}}\right) f^{2}\left(n_{y}\right)}{1+\left(\frac{V_{1}+V_{2}}{\pi v_{o}}\right) f^{2}\left(n_{y}\right)}
$$

and $\eta\left(n_{x}\right)$ is Eq. (4.5), replacing $n_{y}$ by $n_{x}$. For the spin degrees of freedom, $\mu\left(n_{y}\right)$ is given by Eq. (4.5) with $V_{1}$ and $V_{2}$ replaced by $-U_{1}$ and $-U_{2}$ respectively, while $\mu\left(n_{x}\right)$ replaces $n_{y}$ by $n_{x}$.

This completes the diagonalization of the boson problem. It is seen that the spectrum is linear in momentum perpendicular to each face, and each will contribute a term linear in temperature to the specific heat. Interactions renormalize this linear term, as in the Luttinger model. 
These transformations can be used to calculate the fermion correlation function, and determine the relevant operators. It is helpful to use the boson-fermion duality on each chain separately, representing for example the fermion operator of Eq. (2.6) by

$$
\begin{aligned}
& \psi_{1 l \sigma}(x)=Z_{l} e^{-\varphi_{1 l \sigma}(x)+i k_{F} x} \\
& \psi_{2 l \sigma}(x)=Z_{l} e^{\varphi_{2 l \sigma}(x)-i k_{F} x}
\end{aligned}
$$

where the "phase" fields are defined by

$$
\begin{aligned}
\varphi_{\alpha l \sigma}(x) & =(2 N)^{-1} \sum_{\vec{n}} n_{x}^{-1} \rho_{\alpha \sigma}(\vec{n}) e^{-i k_{x} x-i p_{y} l} \\
& =2^{-3 / 2} N^{-1} \sum_{\vec{n}} n_{x}^{-1}\left[\rho_{\alpha}(\vec{n})+\sigma \sigma_{\alpha}(\vec{n})\right] e^{-i k_{x} x-i p_{y} l}
\end{aligned}
$$

with $\alpha=1,2, \sigma= \pm 1$, and separation into charge and spin operators has been used. A renormalization constant $Z_{l}$ has been introduced, which contains the contributions from zero modes corresponding to the $n_{x}=0$ term in Eq. (4.7). For the purely $1 \mathrm{D}$ case $6, Z=(2 \pi \alpha)^{-\frac{1}{2}}$, but for chains, this can also contain a phase factor $\exp \left(i \phi_{\alpha \sigma}(l)\right)$ corresponding to a uniform fermion phase on different chains.

The corresponding operators for the 3-4 faces are defined by

$$
\begin{aligned}
& \psi_{3 k \sigma}(y)=Z_{k} e^{-\varphi_{3 k \sigma}(y)+i k_{F} y} \\
& \psi_{4 k \sigma}(y)=Z_{k} e^{\varphi_{4 k \sigma}(y)-i k_{F} y}
\end{aligned}
$$

and these "phase" fields are found from Eq. (4.7) by the replacements $1 \rightarrow 3,2 \rightarrow 4$, and $n_{x}$ by $n_{y}$. For these 3-4 face operators, $y$ is now the continuum valuable, and $k$ represents the discrete $x$-variable. Thus the plane wave phase factor $k_{x} x+p_{y} l$ in Eq. (4.7) is replaced by $k_{y} y+p_{x} k$, for the $\varphi_{\alpha k \sigma}(y)$ fields, for $\alpha=3,4$.

In the following, it will be shown that fermion operator must be paired on equal chains, otherwise the corresponding terms are irrelevant. That simplifies the zero mode factors, $Z$, since products of fermion operators on different chains commute, and potential single fermion ordering terms drop out.

The calculation of a fermion correlation function in the boson ground state separates into products of operators in the spin and charge sectors, as seen by the example

$$
\begin{aligned}
\left\langle\psi_{1 l+}^{\dagger}(x, t) \psi_{10+}\right\rangle & =Z^{2}\left\langle e^{\varphi_{1 l+}(x t)} e^{\left.-\varphi_{10+}\right\rangle e^{-i k_{F} x}}\right. \\
& =Z^{2} e^{-i k_{F} x} A_{l}(x, t) B_{l}(x, t)
\end{aligned}
$$

where 


$$
A_{l}(x, t)=\left\langle e^{N^{-1} 2^{-\frac{3}{2}} \sum_{\vec{n}} n_{x}^{-1} \rho_{1}(\vec{n}, t) e^{-i k_{x} x-i p_{y} l}} e^{-N^{-1} 2^{-\frac{3}{2}} \sum_{\vec{n}} n_{x}^{-1} \rho_{1}(\vec{n})}\right\rangle
$$

with time evolution, and expectation values using the $\rho$ part of the Hamiltonian in Eq. (4.3). In identical manner, the $B$ term replaces $\rho_{1}$ with $\sigma_{1}$, and uses the $\sigma$ part of Eq. (4.3).

Diagonalizing the Hamiltonian mixes $\rho_{1}$ and $\rho_{2}$ according to Eq. (4.2), such that $A$ is a product $A_{1} A_{2}$ involving the " 1 " and " 2 " operators

$A_{1_{l}}(x, t)=\left\langle e^{N^{-1} 2^{-\frac{3}{2}} \sum_{n} n_{x}^{-1} \cosh \eta\left(n_{y}\right) \rho_{1}(\vec{n}, t) e^{-i k_{x} x-i p_{y} l}} e^{-N^{-1} 2^{-\frac{3}{2}} \sum_{n} n_{x}^{-1} \cosh \eta\left(n_{y}\right) \rho_{1}(\vec{n})}\right\rangle$

with $A_{2}(x, t)$ given by replacing $\cosh \eta\left(n_{y}\right)$ by $\sinh \eta\left(n_{y}\right)$ and $\rho_{1}$ by $\rho_{2}$.

In this expectation value, the time evolution $\rho_{1}(\vec{n}, t)=\rho_{1}(\vec{n})=e^{i k_{x} v_{o} \gamma\left(n_{y}\right) t}$ is to be used. These can be calculated using the Baker-Hausdorf formula to find

$A_{1 l}(x, t)=\exp \left\{-(4 N)^{-1} \sum_{n_{y}=-N}^{N} \sum_{n_{x}=1}^{\infty} n_{x}^{-1} \cosh ^{2} \eta\left(n_{y}\right) e^{-\alpha k_{x}}\left(1-e^{i p_{y} l+i k_{x}\left(x-v_{o} \gamma\left(n_{y}\right) t\right)}\right)\right\}$

with $A_{2 l}(x, t)$ replacing $x$ by $-x$ and $\cosh ^{2} \eta\left(n_{y}\right)$ by $\sinh ^{2} \eta\left(n_{y}\right)$. Converting the sum over $n_{x}$ to an integral over $k_{x}$, it is seen that this integral diverges to $(-\infty)$ at $k_{x}=0$, unless $l=0$. Thus, at $t=0$ :

$$
A_{1 l}(x, t)=\delta_{l, o}\left(\frac{i \alpha}{x+i \alpha}\right)^{\frac{1+d \rho}{2}}
$$

where $d_{\rho}=N^{-1} \int_{o}^{N} d n_{y} \cosh ^{2} \eta\left(n_{y}\right)$. The time dependence is complicated by the dependence of velocity on $n_{y}$ but as long as it remains positive, Eq. (4.11) provides a calculation of the operator dimension of $A_{1}$, since $t$ scales as $x$. Proceeding to the other expectation values, there results:

$$
\begin{aligned}
& A_{2 l}(x)=\delta_{l, 0}\left(\frac{i \alpha}{-x+i \alpha}\right)^{\frac{d_{\rho}}{2}} \\
& B_{1 l}(x)=\delta_{l, 0}\left(\frac{i \alpha}{x+i \alpha}\right)^{\frac{1+d_{\sigma}}{2}} \\
& B_{2 l}(x)=\delta_{l, 0}\left(\frac{i \alpha}{-x+i \alpha}\right)^{\frac{d_{\sigma}}{2}}
\end{aligned}
$$

and consequently 


$$
\left\langle\psi_{1 l+}^{\dagger}(x) \psi_{10+}\right\rangle=\delta_{l, 0} e^{-i k_{F} x}\left(\frac{1}{2 \pi \alpha}\right)\left(\frac{i \alpha}{x+i \alpha}\right)\left(\frac{\alpha^{2}}{x^{2}+\alpha^{2}}\right)^{\frac{d_{\rho}+d_{\sigma}}{2}}
$$

where $d_{\sigma}$ is found as in Eq. (4.12) for the spin degrees of freedom replacing $\eta$ by $\mu$. This is the result of independent chains of the Luttinger model with a suitably chosen coupling constant. The time dependence is more complicated since the boson spectrum depends on $n_{y}$, but the essential physics remains $1 \mathrm{D}$ in character.

The occupation number for an electron $\left\langle n_{1 l \sigma}\left(k_{x}\right)\right\rangle$ on chain $l$ is an integrall over the correlation function of Eq. (4.13). It is seen that only fermion operators on the same chain contribute, so the occupation number is independent of $l$. Since the $x$ dependence of Eq. (4.13) is the same as in the Luttinger model, it can be concluded that $\left\langle n_{1 l \sigma}\left(k_{x}\right)\right\rangle$ is continuous at $k_{x}=k_{F}$. For other faces, the result is identical. Bosonic portions of the interactions on a square Fermi surface destroy Fermi-liquid behaviors.

It is now appropriate to check the assumption of Eq. (2.14) concerning the off-diagonal matrix elements of $t_{l, l^{\prime}}$, using the boson problem of Eq. (4.3) as the starting point. This is done using perturbation theory in $t_{l, l^{\prime}}$ to check whether this operator is relevant. One of the terms to be studied is

$$
T_{1 \sigma}(x)=\sum_{l \neq l^{1}} t_{l l^{\prime}} \psi_{1 l \sigma}^{\dagger}(x) \frac{\partial}{\partial x} \psi_{1 l^{\prime} \sigma}(x)
$$

and in second order perturbation theory would contribute

$$
\int d x \int d t \int d x^{\prime} \int d t^{\prime}\left\langle T_{1 \sigma}(x, t) T_{1 \sigma}\left(x^{\prime}, t^{\prime}\right)\right\rangle
$$

where time evolution and averaging is with respect to $H_{o}+H_{B}$.

Using the boson representation for the $\psi_{\alpha l \sigma}(x)$ operator, the expectation value in Eq. (4.14) is calculated to be proportional to:

$$
\sum_{l_{1} \neq l_{2}} t_{l_{1}-l_{2}}^{2} \frac{1}{\left(R-V^{\prime} \tau+i \alpha\right)^{4}}\left(\frac{\alpha^{2}}{R^{2}-V^{\prime 2} \tau^{2}+\alpha^{2}}\right)^{d\left(l_{1}-l_{2}\right)}
$$

where $V^{\prime}$ is an average (finite) velocity, instead of doing the integral over $\gamma\left(n_{y}\right), R=x-$ $x^{\prime}, \tau=t-t^{\prime}$, and $d\left(l_{1}-l_{2}\right)$ is given by:

$$
d\left(l_{1}-l_{2}\right)=N^{-1} \int_{o}^{N} d n_{y}\left[\sinh ^{2} \eta\left(n_{y}\right)+\sinh ^{2} \mu\left(n_{y}\right)\right]\left[1-\cos p_{y}\left(l_{1}-l_{2}\right)\right]
$$

For large $l_{1}-l_{2}$, the cosine term gives a vanishing contribution, and Eq. (4.15) reduces to the expected result from naive dimensional analysis, using Eq. (4.12), with $d(\infty)=d_{\rho}+d_{\sigma}$.

Since $d\left(l_{1}-l_{2}\right)>0$, the integrals over space-time in Eq. (4.15) are convergent at large 
distance giving a factor of the two dimensional volume. The sum over $l_{1}$ and $l_{2}$ is proportional to $N$, since $t_{l_{1}-l_{2}}$ falls off as $\left(l_{1}-l_{2}\right)^{-2}$. This result means $T_{1 l_{1} \sigma}(x)$ is an irrelevant perturbation, the usual result in two space-time dimensions, when the operator dimension is greater than two. The reduction from two to one space dimensions is a consequence of the finite sum over chains, a result reminiscent of independent one dimensional chains.

In similar fashion, the contributions from the other operators can be seen to be irrelevant. Consequently, the modulation of the Fermi velocity along the face of the Fermi surface is an irrelevant perturbation about the boson Hamiltoniand. This result should be contrasted with perturbation theory about the free fermion Hamiltonian, which contains $(\log )^{2}$ singularities resulting from the vanishing Fermi velocity at the corners of the square Fermi surface.

Any non-zero interaction will lead to the irrelevancy of the Fermi velocity modulation, but a much more restrictive condition results for the stability of the square Fermi surface itself, away from half-filling. The appropriate perturbation is the number operator which is given for the " 1 " face by:

$$
\Delta \mu N_{1 \sigma}=\Delta \mu\left(\pi k_{F}^{-1}\right) \int d x \sum_{l, l_{1} l_{2}} g\left(l-l_{1}\right) g\left(l-l_{2}\right) \psi_{1 l_{1} \sigma}^{\dagger}(x) \psi_{1 l_{2} \sigma}(x)
$$

where $\Delta \mu$ is the change in chemical potential from half-filling. The corresponding correlation function to second order is proportional to:

$$
\sum_{l, l_{1}, l_{2}} g^{2}\left(l-l_{1}\right) g^{2}\left(l-l_{2}\right) \frac{1}{\left(R-V^{\prime} \tau+i \alpha\right)^{2}}\left(\frac{\alpha^{2}}{R^{2}-V^{\prime 2} \tau^{2}+\alpha^{2}}\right)^{d\left(l_{1}-l_{2}\right)}
$$

since the two derivative operators are absent. For this to be irrelevant, $d\left(l_{1}-l_{2}\right)>2$ for all $l_{1}-l_{2}$, as discussed above. This requires a sufficiently strong interaction to be present. Under this circumstance, the only contribution is the diagonal term, $l_{1}=l_{2}$, given by

$$
N_{1 \sigma}(x)=\frac{\pi}{2 k_{F}} \sum_{l_{1}} \rho_{1 l, \sigma}(x)
$$

where the factor of $1 / 2$ comes from $\sum_{l} g^{2}\left(l-l_{1}\right)$. The presence of this term simply shifts the Fermi momentum uniformly, the square Fermi surface remains a square.

Strong interactions tend to localize single electrons to a chain, at least in lowest order. In higher orders, or when interactions between adjacent faces are included, it is possible that the situation might change. It seems intuitively plausible that a crossover from a square to a rounded Fermi surface should occur, but it is not yet clear which terms are responsible for this. Intra-facial hopping terms, such as $\psi_{1}^{\dagger} \psi_{3}$, etc, would lead to rounding. These do not appear in lowest order, but could enter in connection with some of the relevant operators discussed in the following section. This problem requires further attention. It is nonetheless very interesting that the square Fermi surface is stable at this level. 


\section{RELEVANT INTERACTIONS}

Interactions between chains are generated through the mapping of the fermion operators for the four faces onto independent chains. As described in the previous section, it is possible to extract a purely bosonic interaction, and use this to define a new starting point for perturbation theory. Other interactions remain, and it is necessary to construct a new interaction Hamiltonian, and attempt to solve this new problem. This situation is similar to the Kondo problem or the backward scattering model, where a similar reconstruction of the interactions is possible, leading to a new expansion.

Consider the interactions involving parallel faces, the "1" and "2" operators. The lattice term $U \sum_{i} n_{i+} n_{i-}$ would contain

$$
\sum_{i} n_{i+} n_{i-} \rightarrow \sum_{l} \int d x \Psi_{l+}^{\dagger}(x) \Psi_{l,+}(x) \Psi_{1,-}^{\dagger}(x) \Psi_{l,-}(x)
$$

with the $\Psi_{l, \sigma}(x)=\psi_{1 l \sigma}^{\prime}(x)+\psi_{2 l \sigma}^{\prime}(x)$, and these primed operators are, in turn, sums over independent chains given by Eq. (2.6). There are similar terms for the " 3 " and " 4 " faces, and cross terms containing interactions between adjacent faces. Cross terms are not considered here. The task now is to extract the purely boson interaction terms in the sum, giving $H_{B}$ of the previous sections, and find the remaining operators. These new operators then provide the desired perturbation expansion.

A conclusion of the previous section is that the sum over independent chain Fermi operators must have chain indices paired. If a single unpaired index appears, that operator will contribute an operator dimension greater than 1/2, as in Eq. (4.13), and the remaining three greater than $3 / 2$, giving an overall dimension greater than 2 . In the second order correlation function using Eq. (5.1), the overall dimension will be greater than 4, and hence is an irrelevant term. This is another manifestation of the irrelevance of single particle hopping for the square Fermi surface.

Consider first the terms which occur when fermion operators with the same spin are paired on the same chain. If they have the same face index, there are boson operators already included in $H_{B}$. Otherwise, there are two types of terms

$$
\begin{aligned}
H_{B S} & =U \pi^{2} k_{F}^{-2} \int d x \sum_{l_{1} l_{2}} G\left(l_{1}-l_{2}\right)\left[\psi_{1 l_{1}+}^{\dagger}(x) \psi_{2 l_{1}+}(x) \psi_{2 l_{2}-}^{\dagger}(x) \psi_{1 l_{2}-}(x)+\text { h.c. }\right] \\
H_{U} & =U \pi^{2} k_{F}^{-2} \int d x \sum_{l_{1} l_{2}} G\left(l_{1}-l_{2}\right)\left[\psi_{1 l_{1}+}^{\dagger}(x) \psi_{2 l_{1}+}(x) \psi_{1 l_{2}-}^{\dagger}(x) \psi_{2 l_{2}-}(x)+\text { h.c. }\right]
\end{aligned}
$$

where

$$
G\left(l_{1}-l_{2}\right)=\sum_{l} g^{2}\left(l-l_{1}\right) g^{2}\left(l-l_{2}\right)=(12)^{-1} \delta_{l_{1}, l_{2}}+2^{-1} \pi^{-2}\left(l_{1}-l_{2}\right)^{-2} .
$$


Physically, $H_{B S}$ describes backward scattering on same or different chains, while $H_{U}$ is an Umklapp process.

In a $1 \mathrm{D}$ system, it is known that $H_{U}$ is relevant and $H_{B S}$ is irrelevant for repulsive interaction 3 , while these roles are reversed for attractive interactions. The situation is similar here, as can be shown using the fermion-boson duality principle from the previous section.

The Umklapp term for $l_{1}=l_{2}$ in the boson representation is given by:

$$
H_{U}=-U G(0)\left(2 k_{F} \alpha\right)^{-2} \sum_{l} \int d x\left[e^{-4 i k_{F} x} e^{-\sqrt{2}\left(\varphi_{1 \rho l}(x)+\varphi_{2 \rho l}(x)\right)}+\mathrm{hc}\right]
$$

where the $\varphi_{1 \rho l}$ operators are defined in Eq. (4.6). $H_{B S}$ is the same with the $\varphi_{1 \rho l}$ operators replaced by $\varphi_{1 \sigma l}$ operators, and the $e^{4 k_{F} x}$ phase factor is absent. The minus sign results from ordering Eq. (5.3) into the form $(-) \psi_{1 l+}^{\dagger} \psi_{2 l-} \psi_{1 l_{-}}^{\dagger} \psi_{2 l+}$. It can then be confirmed that the matrix element of this operator between the states $\left\langle\psi_{2 l_{1}-}^{\dagger}\left(x_{2}\right) \psi_{1 l_{1}+}\left(x_{2}\right)\right|$ and $\left.\psi_{2 l_{1}+}^{\dagger}\left(x_{1}\right) \psi_{1 l_{1}-}\left(x_{1}\right)\right\rangle$ is the same as the boson equivalent in Eq. (5.4), sandwiched between the boson equivalents of these two states.

Diagonalizing $H_{o}+H_{B}$ as in section IV with $S_{1}$, will transform the exponent in Eq. (5.4) according to

$$
\begin{aligned}
e^{S_{1}} & (2 N)^{-1} \sum_{\vec{n}} n_{x}^{-1}\left(\rho_{1}(\vec{n})+\rho_{2}(\vec{n})\right) e^{-i k_{x} x-i p_{y} l} e^{-S_{1}} \\
& =(2 N)^{-1} \sum_{\vec{n}} n_{x}^{-1} e^{-\eta\left(n_{y}\right)}\left(\rho_{1}(\vec{n})+\rho_{2}(\vec{n})\right) e^{-i k_{x} x-i p_{y} l}
\end{aligned}
$$

from which the scaling dimension of the Umklapp operator, $d_{U}$ is seen to be

$$
d_{U}=\frac{1}{2 N} \sum_{n_{y}-N}^{N}\left(2 e^{-2 \eta\left(n_{y}\right)}\right)
$$

as discussed in the previous section. This means the correlation function appearing in second order using Eq. (5.3) falls off as (distance) ${ }^{-2 d_{U}}$. The zero interaction case, $\eta=0$, has $2 d_{U}=4$ and for repulsive interactions $d_{U}<2$ from Eq. (4.5). This term is then relevant and must be retained.

The phase factor $e^{4 i k_{F} k}$ for half-filling is evaluated to find $4 k_{F} x=4\left(\pi 2^{-1 / 2} s^{-1}\right)\left(s 2^{-1 / 2}\right)(m+$ $n)=0(\bmod 2 \pi)$ and makes no contribution. Notice that for strong interactions, such that the Fermi surface remains square as discussed in the previous section $k_{F}$ shifts away from $\pi s^{-1} 2^{-\frac{1}{2}}$ under doping and the Umklapp term describes a (quantum) commensurateincommensurate transition 15 . This transition will differ somewhat from the one dimensional problem due to the parallel momentum dependence contained in $H_{B}$. 
Using the diagonalizing transformation for the spin degrees of freedom contained in Eq. (5.2), the operator dimension for equal chain backward scattering is given by

$$
d_{B S}=\frac{1}{2 N} \sum_{n_{y}=-N}^{N}\left(2 e^{-2 \mu\left(n_{y}\right)}\right)
$$

and Eq. (4.5) shows $d_{B S}>2$ for repulsive interactions. For attractive interactions, the roles are reversed, $d_{U}>2$ and $d_{B S}<2$.

When $l_{1} \neq l_{2}$ in Eq. (5.3), the scaling dimension is changed, becoming dependent on $\left(l_{1}-l_{2}\right)$ through the $k_{y}$ dependence in $H_{B}$. In addition, the spin degrees of freedom do not cancel in the exponent when the fermion operators are bosonized and contribute to the exponent of the power law. The result for the operator scaling dimension is found to be:

$$
\begin{array}{r}
d_{U}\left(l_{1}-l_{2}\right)=\frac{1}{2 N} \sum_{n_{y}=-N}^{N}\left[e^{-2 \eta\left(n_{y}\right)}\left(1+e^{i p_{y}\left(l_{1}-l_{2}\right)}\right)\right. \\
+e^{-2 \mu\left(n_{y}\right)}\left(1-e^{i p_{y}\left(l_{1}-l_{2}\right)}\right]
\end{array}
$$

For repulsive interactions, $\eta>0$ and $\mu<0$, and the value of $d\left(l_{1}-l_{2}\right)$ is not necessarily small. The result for $d_{B S}\left(l_{1}-l_{2}\right)$ is found from Eq. (5.7) by interchanging $\eta$ and $\mu$.

At this stage of the analysis, it is helpful to reflect on the significance of the transformations which change the operator dimensions, as determined by $\eta$ and $\mu$. For general repulsive interactions, $\eta>0$ and $\mu<0$, from Eq. (4.5). For these signs, and sufficiently large magnitudes, $d_{B S}\left(l_{1}-l_{2}\right)>2$ for all $l_{1}-l_{2}$, and $d_{U}\left(l_{1}-l_{2}\right)>2$ for $l_{1} \neq l_{2}$. These terms are irrelevant. The surviving relevant operator from this pairing is given by chain diagonal Umklapp processes, with dimension $d_{U}<2$ from Eq. (5.6).

With the coupling constants chosen to lie in this range, it is possible to anticipate the existence of a solution. This will be constructed by dropping the irrelevant operators, and expanding the relevant operators in the small parameters, $e^{-2 \eta}$ or $e^{2 \mu}$. There is a similarity here to the 1D massive Thirring model, where expanding the mass term when the analogous parameter is small, leads to the Klein-Gordon equation 10 .

It is necessary to examine other pairings of Eq. (5.1) to extract further relevant operators. Pairings which involve the first and third terms (with second and fourth also paired) contain terms like

$\psi_{1 l_{1}+}^{\dagger} \psi_{2 l_{1}-}^{\dagger} \psi_{2 l_{2}-} \psi_{1 l_{2}+}, \psi_{1 l_{1}+}^{\dagger} \psi_{2 l_{1}-}^{\dagger} \psi_{1 l_{2}-} \psi_{2 l_{2}+}$, and $\psi_{1 l_{1}+}^{\dagger} \psi_{1 l_{1}-}^{\dagger} \psi_{2 l_{2}-} \psi_{2 l_{2}+}$. After bosonizing these, it is found that they are all irrelevant. The first two give the operator dimension of Eq. (5.8), but with $\eta \rightarrow-\eta$ and $\mu \rightarrow-\mu$, and these are larger than two in the coupling constant region of interest. The third resembles single particle hopping with an extra $\sqrt{2}$ in the exponent after bosonization, and is irrelevant.

Cross terms which leave a factor of $e^{2 i k_{F} x}$ after bosonization can be neglected since they 
have a momentum transfer of $2 k_{F}$. This would require a large energy transfer and the intermediate states would lie far away from the Fermi surface.

The remaining pairing, with the first and fourth terms on the same chain together with the second and third, contains relevant operators. These are given by $-\psi_{1 l_{1}+}^{\dagger} \psi_{2 l_{1}-} \psi_{1 l_{2}-}^{\dagger} \psi_{2 l_{2}+}$ and $-\psi_{1 l_{1}+}^{\dagger} \psi_{2 l_{1}-} \psi_{2 l_{2}-}^{\dagger} \psi_{1 l_{2}+}$, plus the $(1 \leftrightarrow 2)$ interchange. Terms such as $\psi_{1 l_{1}+}^{\dagger} \psi_{1 l_{1}-} \psi_{2 l_{2}-}^{\dagger} \psi_{2 l_{2}+}$ are irrelevant, and those involving $e^{2 i k_{F} x}$ after bosonization can be discarded as previously discussed.

Both relevant operators involve products of $2 k_{F}$ spin-flip excitations on different chains. (The $l_{1}=l_{2}$ terms has already been counted in the first pairing, characterized by $d_{U}, d_{B S}$ and a boson term). After bosonizing, these terms give respectively:

$$
\begin{aligned}
& H_{D U}=-U\left(2 k_{F} \alpha\right)^{-2} \int d x \sum_{l_{1} \neq l_{2}} G\left(l_{1}-l_{2}\right)\left\{e^{\left.\varphi_{\rho l_{1}}(x)+\varphi_{\rho l_{2}}(x)+\tilde{\varphi}_{\sigma l_{1}}(x)-\tilde{\varphi}_{\sigma l_{2}}(x)+(1 \leftrightarrow 2)\right\}}\right. \\
& H_{D B}=-U\left(2 k_{F} \alpha\right)^{-2} \int d x \sum_{l_{1} \neq l_{2}} G\left(l_{1}-l_{2}\right)\left\{e^{\left.\varphi_{\rho l_{1}}(x)-\varphi_{\rho l_{2}}(x)+\tilde{\varphi}_{\sigma l_{1}}(x)-\tilde{\varphi}_{\sigma l_{2}}(x)+(1 \leftrightarrow 2)\right\}}\right.
\end{aligned}
$$

Here, $\sqrt{2} \varphi_{\rho l}(x)=\varphi_{1 \rho l}(x)+\varphi_{2 \cdot \rho l}$ and $\sqrt{2} \tilde{\varphi}_{\sigma l}=\varphi_{1 \sigma l}(x)-\varphi_{2 \sigma l}(x)$ with fields $\varphi_{1 \sigma l}(x)$, etc, defined in Eq. (4.6). It should be noted that $\varphi_{\rho l}(x)$ involves the combination $\left[\rho_{1}(\vec{n})+\rho_{2}(\vec{n})\right]$ which is multiplied by $e^{-\eta\left(n_{y}\right)}$ under diagonalization, as in Eq. (5.5). Similarly, the $\tilde{\varphi}_{\sigma l}(x)$ involves $\left(\sigma_{1}(\vec{n})-\sigma_{2}(\vec{n})\right)$ which is multiplied by $e^{\mu\left(n_{y}\right)}$ under diagonalization, from Eq. (5.5). Both multiplicative factors should be small for the corresponding operator to be relevant. Note that the fermion $(1 \leftrightarrow 2)$ interchange requires $\varphi_{\rho l}$ to change sign, since there is a sign difference in the boson representation of $\psi_{1}$ and $\psi_{2}$, while $\tilde{\varphi}_{\sigma l}$ does not change sign. Under hermitian conjugation both change sign.

It is appropriate to label the first of these terms as "different chain Umklapp", $H_{D U}$, while the second is a "different chain backward scattering", $H_{D B}$. Their scaling dimensions, after diagonalizing $H_{B}$, are given by

$$
\begin{array}{r}
d_{D U}\left(l_{12}\right)=\frac{1}{2 N} \sum_{n_{y}=-N}^{N}\left[e^{-2 \eta\left(n_{y}\right)}\left(1+e^{i p_{y} l_{12}}\right)\right. \\
\left.+e^{2 \mu\left(n_{y}\right)}\left(1-e^{i p_{y} l_{12}}\right)\right] \\
d_{D B}\left(l_{12}\right)=\frac{1}{2 N} \sum_{n_{y}=-N}^{N}\left[e^{-2 \eta\left(n_{y}\right)}\left(1-e^{i p_{y} l_{12}}\right)\right. \\
\left.+e^{2 \mu\left(n_{y}\right)}\left(1-e^{i p_{y} l_{12}}\right)\right]
\end{array}
$$


Both are relevant (dimension less than two) for $\eta>0$ and $\mu<0$, the repulsive case of interest. There is a region of parameter space where all three relevant operators have a small dimension. In this case, the exponents contained in Eq. (5.4), Eq. (5.9), and Eq. (5.10) can be expanded.

Scattering between parallel faces results in an equivalence to interacting fermions on separate chains. In this section, the interaction terms have been extracted and their scaling dimensions calculated. There is a strong coupling region, characterized by three operators with small dimension, $d_{U}, d_{B U}$ and $d_{D B}$, which must be included. The following section presents the construction of the solution.

\section{GAP GENERATION AND THE INSULATING PHASE}

This section begins with the boson problem of section III, adding the terms with relevant operators from the previous section. It would be interesting to find a solution for this problem, however this has not been found for general coupling constants. But there is a limit where a solution is possible for particular choices of coupling constants. This choice corresponds to the parameters $e^{-2 \eta\left(n_{y}\right)}$ and $e^{2 \mu\left(n_{y}\right)}$ small, such that the exponents of Eq. (5.4), Eq. (5.9) and Eq. (5.10) can be expanded. Physically, this is a strong repulsion regime, with some restrictions on the spin dependent interactions, $U_{1}$ and $U_{2}$ of section III.

The boson part of the problem, $H_{o}+H_{B}$ is given by Eq. (4.1). From the preceding section, the three relevant operators must be added to define a new "interaction" term. Diagonalizing $H_{o}+H_{B}$, using the $S$ operators of Eq. (4.2), transforms the "phase" fields appearing in the relevant operators, generally decreasing them (for $e^{-\eta}$ and $e^{\mu}$ both small). Expanding

these phase factors to quadratic order gives the new interaction Hamiltonian. For the 1-2 faces, this is:

$$
\begin{aligned}
& H=H_{D}+H_{\mathrm{int}} \\
& H_{\mathrm{int}}=-2 U\left(2 k_{F} \alpha\right)^{-2} \int d x \sum_{l_{1}, l_{2}} G\left(l_{1}-l_{2}\right) e^{S}\left[\varphi_{\rho l_{1}}^{2}(x)+\varphi_{\rho l_{2}}^{2}(x)+\left(\tilde{\varphi}_{\sigma l_{1}}-\tilde{\varphi}_{\sigma l_{2}}\right)^{2}\right] e^{-S}
\end{aligned}
$$

where $H_{D}$ is Eq. (4.3) and $S=S_{1}+S_{2}$ from Eq. (4.2). Not only do the first order terms cancel, but the cross terms between spin and charge operators cancel as well. To this order, the Hamiltonian separates into independent spin and charge sectors.

After Fourier transforming, the new Hamiltonian can be written in terms of the density operators as $H=H_{\rho}+H_{\sigma}$, where:

$$
\begin{aligned}
& H_{\rho}=\frac{\pi v_{o}}{2 N L} \sum_{\vec{n}}\left\{\gamma\left(n_{y}\right)\left[\rho_{1}(\vec{n}) \rho_{1}(-\vec{n})+\rho_{2}(\vec{n}) \rho_{2}(-\vec{n})\right]\right. \\
& \left.+M_{\rho}^{2}\left(n_{y}\right) k_{x}^{-2}\left[\rho_{1}(\vec{n})+\rho_{2}(-\vec{n})\right]\left[\rho_{1}(-\vec{n})+\rho_{2}(-\vec{n})\right]\right\}
\end{aligned}
$$




$$
\begin{array}{r}
H_{\sigma}=\frac{\pi v_{o}}{2 N L} \sum_{\vec{n}}\left\{\delta\left(n_{y}\right)\left[\sigma_{1}(\vec{n}) \sigma_{1}(-\vec{n})+\sigma_{2}(\vec{n}) \sigma_{2}(-\vec{n})\right]\right. \\
\left.+M_{\sigma}^{2}\left(n_{y}\right) k_{x}^{-2}\left[\sigma_{1}(\vec{n})+\sigma_{2}(\vec{n})\right]\left[\sigma_{1}(-\vec{n})+\sigma_{2}(-\vec{n})\right]\right\}
\end{array}
$$

with velocity renormalizations given by Eq. (4.4), and

$$
\begin{aligned}
& M_{\rho}^{2}\left(n_{y}\right)=\frac{8 \pi v_{o} U \gamma\left(n_{y}\right)}{\left(k_{F} \alpha\right)^{2}} e^{-2 \eta\left(n_{y}\right)} f^{2}(0) \\
& M_{\sigma}^{2}\left(n_{y}\right)=\frac{4 \pi v_{o} U \delta\left(n_{y}\right)}{\left(k_{F} \alpha\right)^{2}} e^{2 \mu\left(n_{y}\right)}\left[f^{2}(0)-f^{2}\left(n_{y}\right)\right]
\end{aligned}
$$

Here $\eta$ and $\mu$ are given by Eq. (4.5) and $f^{2}\left(n_{y}\right)$ under Eq. (3.6). The combinations in these $M^{2}$ are chosen such that the spectrum of Eq. (6.2) and Eq. (6.3) takes the "relativistic" form

$$
\begin{aligned}
& E_{\rho}^{2}(\vec{n})=k_{x}^{2} v_{o}^{2} \gamma^{2}\left(n_{y}\right)+M_{\rho}^{2}\left(n_{y}\right) \\
& E_{\sigma}^{2}(\vec{n})=k_{x}^{2} v_{o}^{2} \delta^{2}\left(n_{y}\right)+M_{\rho}^{2}\left(n_{y}\right)
\end{aligned}
$$

as can easily be confirmed by diagonalizing these two. The contributions from the 3-4 faces result in an identical Hamiltonian, with $\eta_{x} \leftrightarrow n_{y}$.

It is clear that the charge density gap is only weakly dependent on the transverse momentum, through the velocity renormalization. On the other hand, the spin density gap squared vanishes at $n_{y}=0$, and increases linearly with momentum. A quadratic dependence on $n_{y}$ would have been intuitively understandable, since it could be renormalized to give an isotropic excitation spectrum, proportional to $n_{x}^{2}+n_{y}^{2}$. Perhaps the inclusion of interactions between adjacent faces could change $\left|n_{y}\right|$ to $n_{y}^{2}$, but that remains unclear at present.

Renormalization of the bare coupling constant $U$ in Eq. (6.1) is required, if the limit $\alpha \rightarrow 0$ is to be taken, as in the purely $1 \mathrm{D}$ problem 8 . Alternatively, one can regard the $M^{2}$ quantities, as physically observable, setting a length scale for which the bare parameters are determined.

This completes the present analysis of interacting electrons on a square Fermi surface. It focussed on interactions between parallel faces, although the method can be readily generalized to adjacent face interactions. Perhaps the most interesting result concerns the mapping onto chains, and the ability to extract relevant operators in the chain picture.

It should be possible to study this mapping more systematically using renormalization group scaling. If each non-boson operator is assigned a coupling constant, as in $1 \mathrm{D}$ systems 3 , these will satisfy scaling equations. Presumably, some will scale to stronger coupling strengths, and solutions such as those presented here, could characterize the asymptotic behavior. It will be interesting to confirm, or disprove, the strong coupling region outlined here. 
Doping away from this point will generate a phase factor in the Umklapp terms, $H_{U}$ and $H_{D U}$, but not in $H_{D B}$. This phase factor of $\exp \left(4 i \delta k_{F} x\right)$, where $\delta k_{F}$ is the departure from the half-filled value, changes the nature of the problem. The low energy excitations then include some charge states, and it might be expected that a new type of generalized Luttinger model results.

Spin excitations are more problematic in this solution. Although the spin excitation energy vanishes when $p_{y} \rightarrow 0$, it does not vanish linearly. At $k_{x}=0$, the spectrum is proportional to $\left|p_{y}\right|^{1 / 2}$. It is not clear if this is an artifact of parallel face interactions and will be modified by adjacent face scattering. Another possibility is contained in the problem of uniform phase ordering in the chain mapping. As indicated under Eq. (4.7), a uniform phase on each chain is permitted. Perhaps other configurations can be found with lower ground state energy than the present result.

\section{DISCUSSION AND CONCLUSION}

This paper has presented a mapping of the square Fermi surface onto two sets of chains, one set for each axis of the square. It correctly describes electronic excitations near the half-filled band, with the average occupation number near one. Interactions between electrons separate naturally into boson operators on these chains, plus additional operators. The kinetic energy plus the boson operators define a new starting point for perturbation theory, with the additional operators as the perturbation.

This method provides a very powerful new tool for analyzing the properties of the square Fermi surface. The purely bosonic part of the problem can be diagonalized exactly, giving behavior similar to the Luttinger model. It exhibits power low correlations functions and has no jump in the occupation number at the Fermi level. Modulation of the Fermi velocity along the face of the square is irrelevant. Rounding of the square is similarly irrelevant for sufficiently strong interaction strengths.

All of these results are derived using the new perturbation theory about the boson part of the problem. The interactions also contain non-boson terms, which have been analyzed using boson-fermion duality for the chain representations. There is a region of strong coupling when the relevant operator generated by interactions can be solved exactly, in the sense that the sine-Gordon problem becomes the Klein-Gordon problem for strong fermion interaction 10 . For half-filling, a gap appears in the charge spectrum, while the spin excitations remain gapless.

While the mapping works for very general interactions, this paper has concentrated on a special case of parallel face interactions. For a complete solution, interactions between adjacent faces and possible momentum dependences need to be included. A convincing solution of the Hubbard model awaits an analysis of these generalizations. 
When doped away from half-filling, spinless fermion states above the charge gap are occupied. This situation is reminiscent of the commensurate-incommensurate transition 15 , with additional complications due to the transverse momentum dependence. Although the spin degrees of freedom are unchanged, it will be interesting to explore the changes in the charge excitations. In this way, the very interesting issue of superconducting correlations can be resolved.

Lattice degrees of freedom must also be included, and a logical procedure would involve diagonalizing the interesting electron problem first. Adding in the electron-phonon coupling, selecting out the relevant operators, and solving this new problem, would cast new light on the problem of superconductivity in the Cuprate compounds. It seems these steps are all possible within the mapping framework.

It would also seem this problem of interacting fermions on a square Fermi surface could be addressed in other ways. The requirement that fermions must be paired on separate chains could be realized by a gauge symmetry. Since the single chain problem can be solved by a Bethe Ansatz 16 , perhaps this new model of coupled chains has a solution involving superpositions of these single chain Bethe Ansatz hypothesis wavefunctions. It might be simpler to test this on the spinless fermion problem.

Solutions for the problem of interacting electrons on a square Fermi surface, of the type presented here, represent a new regime with correlation effects dominant. Much of the intuition based on Fermi liquid behavior, is no longer applicable. If it can be shown that the high temperature superconductors fall in this regime, this is truly strongly correlated superconductivity. 


\section{REFERENCES}

${ }^{1}$ P. W. Anderson, High Temperature Superconductivity, to be published.

${ }^{2}$ Sudip Chakravarty, Asle Sudbo, Phillip W. Anderson, and Steven Strong, to be published.

${ }^{3}$ J. Solyom, Advances in Physics, 28, 201 (1979).

${ }^{4}$ I. E. Dzyaloshinskii, Zh. Eksp. Teor. Fiz. 931487 (1987) [Sov. Phys. JETP 66, 848 (1987)].

${ }^{5}$ H. J. Schultz, Phys. Rev. B 39, 2940 (1989).

${ }^{6}$ A. Luther and I. Peschel, Phys. Rev. B 12, 3908 (1975).

${ }^{7}$ D. C. Mattis and E. H. Lieb, J. Math. Phys. 6, 3304 (1965).

${ }^{8}$ A. Luther and V. J. Emery, Phys. Rev. Lett. 33, 589, (1974).

${ }^{9}$ P. W. Anderson and G. Yuval, Phys. Rev. Lett. 23, 89 (1969).

10 S. Coleman, Phys. Rev. D 11, 2088 (1975).

${ }^{11} \mathrm{D}$. Schmeltzer, to be published.

${ }^{12}$ Y. Yu, Y. M. Li and N. d'Ambrumenil, to be published.

${ }^{13}$ R. Hlubina, to be published.

14 A. Luther, Phys. Rev. B 14, 2153 (1976).

${ }^{15}$ V. L. Pokrovsky and A. L. Talapov, Phys. Rev. Lett. 42, 65 (1979).

${ }^{16}$ H. Bergknoff and H. B. Thacker, Phys. Rev. D 19, 3666 (1979) 